\section{Highlights from the British Society for Heart Failure 16th Annual Autumn Meeting: 'Making sense of acute heart failure'}

Jane A Cannon ${ }^{* 1} \&$ Jonathan R Dalzell

16th Annual Autumn Meeting of the British Society of Heart Failure: 'Making sense of acute heart failure', London, UK, 28-29 November 2013

The 16th Annual Autumn Meeting of the British Society of Heart Failure entitled 'Making sense of acute heart failure' took place on the 28-29 November 2013 at the Queen Elizabeth II Conference Centre in London. This year saw the 1000th member join the British Society of Heart Failure and over 700 delegates from the UK, Europe and North America attended this year's meeting. Professionals from a range of backgrounds were present including physicians, nurses, scientists, trainees and representatives from industry. The symposium, which is accredited by the Royal College of Physicians and the Royal College of Nursing, highlighted that although we have recently seen substantial progress in the management and outcomes of patients with chronic heart failure, acute heart failure management and outcomes have not changed significantly over almost a generation.

\section{Acute heart failure: trials \& tribulations}

Session one opened with a keynote talk from Professor John McMurray (University of Glasgow, UK) who reviewed the definition of acute heart failure and explained why, perhaps, it is an unhelpful label (Figure 1). Acute and chronic heart failure are not two distinct entities and the overlap between them is a gray area. Despite his involvement in many key acute heart failure trials, he reminded us that the only level 1A evidence-based treatment for acute heart failure is thromboprophylaxis and that if we want to improve outcomes for these patients, further research (with large patient cohorts) is required. Previous acute heart failure trials were reviewed including DOSE, CARESS-HF, ROSE-AHF, RELAX-AHF and ATOMIC-HF. The results from these trials have mainly been disappointing with the notable exception of RELAX-AHF. This recent large, randomized, placebo-controlled trial of serelaxin (recombinant relaxin-2), in addition to standard therapy in patients with acute heart failure, showed a signal towards improvement in patient reported dyspnea and 180-day mortality. These findings have prompted the RELAX-AHF 2 trial which is currently underway and will be adequately powered to definitively delineate the impact serelaxin on mortality.

McMurray pointed out some potentially significant differences between RELAX-AHF and some of the previous acute heart failure trials involving vasodilators (e.g., ASCEND-HF). The differences included the exclusion of patients with a systolic blood pressure of $\leq 125 \mathrm{mmHg}$

'BHF Glasgow Cardiovascular Research Centre, University of Glasgow, 126 University Place, Glasgow, G12 8TA, UK *Author for correspondence: Tel.: +44 141330 2237; Fax: +44 141330 6955; janecannon@nhs.net

\section{KEYWORDS}

- British Society for Heart Failure $\bullet$ heart failure $\bullet$ heart failure preserved ejection fraction 


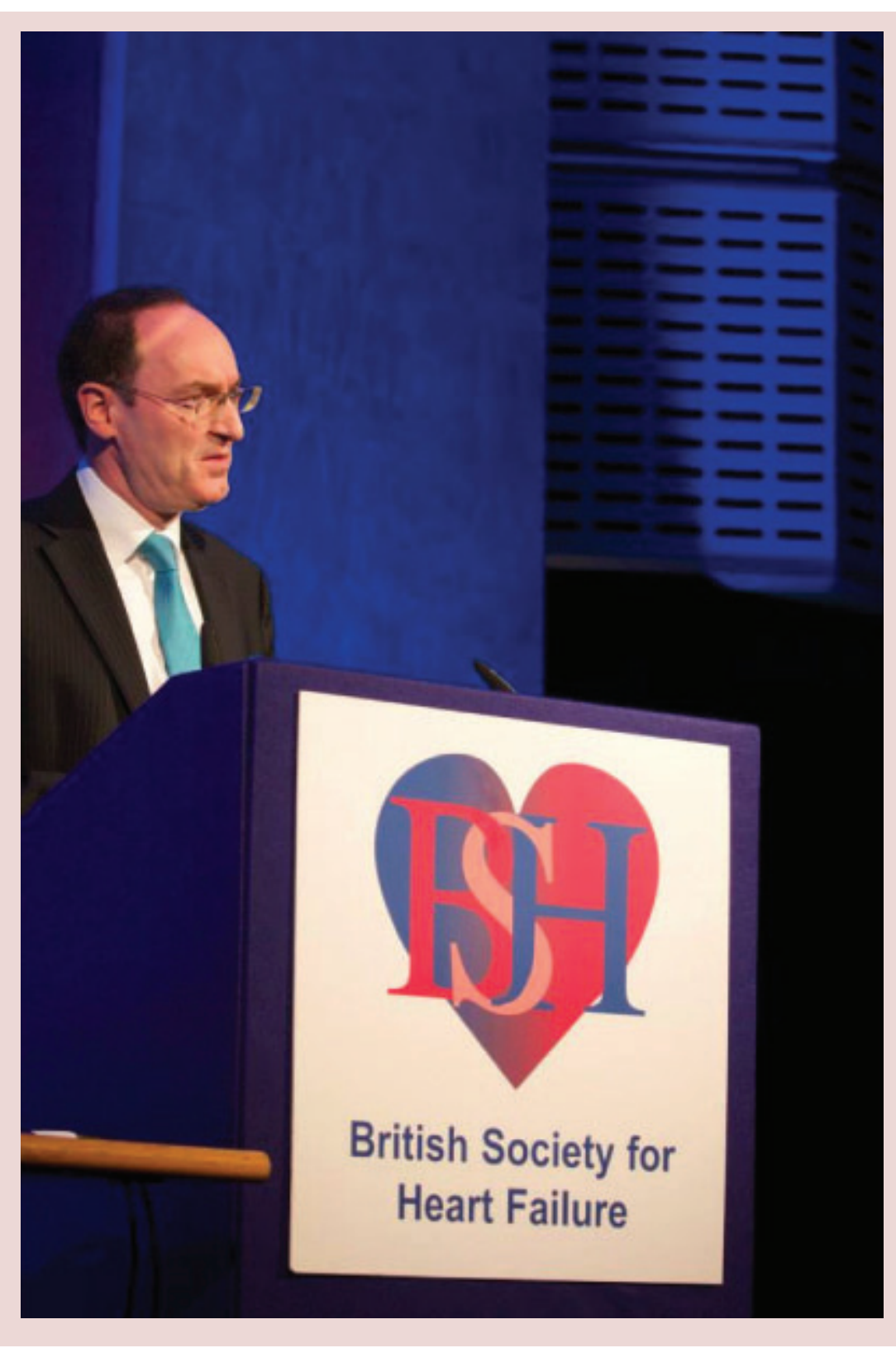

Figure 1. Professor John McMurray (University of Glasgow, UK).

from RELAX-AHF and the fact that the time from presentation to randomization (and study drug initiation) tended to be shorter in RELAX-AHF (within $16 \mathrm{~h}$ of hospitalization with a mean of $7.8 \mathrm{~h}$ ). It may be that earlier treatment could reduce end organ damage and may reduce mortality in a manner similar to thrombolytics in myocardial infarction - could the expression 'time is muscle' be applied to acute heart failure as well?

McMurray also mentioned ularitide, which is a synthetic analog of urodilatin. Urodilatin is an endogenous natriuretic peptide expressed in the kidney. Phase I/II studies of parenteral administration of exogenous ularitide in acute heart failure results in reduction in right atrial pressure, pulmonary wedge pressure and NT-proBNP with no adverse effect on renal function. A large international Phase III trial of ularitide versus placebo in patients with acute heart failure and preserved blood pressure, TRUE-AHF, is currently underway.

Professor John Cleland (Imperial College, London, UK) presented UK data showing that the most common reason for hospitalization of heart failure patients is a combination of other medical comorbidities, with only a minority of patients having a sudden onset of isolated heart failure symptoms with no prodrome (Figure 2). Those presenting with heart failure mainly present with peripheral fluid retention rather than pulmonary edema and are often referred to as 'comfortable at rest but breathless on slight exertion' (CARBOSE), rather than being 'short of breath at rest' (SOBAR). The most common causes of sudden, unheralded worsening heart failure in the UK are the development of atrial fibrillation (with rapid ventricular rate) or acute myocardial infarction.

The National Heart Failure audit was established in 2007 and this year saw the publication of the 6th annual report, based on patients discharged with a diagnosis of heart failure between 1st April 2012 and 31st March 2013. Professor Theresa McDonagh (King's College, London, UK) presented the main findings and recommendations (Figure 3 ). The audit represents $60 \%$ of all heart failure discharges or deaths in England and Wales during this time frame. A total of $66 \%$ of patients were over the age of 75 years, with the majority being male. We learned that over the past year, prescription rates for disease-modifying drugs have increased, with $85 \%$ of patients with left ventricular systolic dysfunction now receiving treatment with angiotensin-converting-enzyme inhibitors or angiotensin receptor blockers. Approximately $60 \%$ of patients are now referred to a heart failure liaison nurse on discharge, suggesting improved service provision compared with previous audit years. In-hospital mortality had a relative risk reduction of $15.3 \%$ and an absolute risk reduction of $1.7 \%$ (from 2011/12-2012/13). This is in keeping with improved implementation of disease-modifying drugs. McDonagh emphasized that improving the standard of care for heart 
failure patients depends on a multidisciplinary approach including healthcare professionals, as well as commissioners using audit data to monitor performance, encourage progress and ensure adequate provision of specialist acute heart failure care.

McDonagh finished by reinforcing the importance of reflecting on our own current practice to identify our weaknesses and so implement changes with the aim of treating patients better and improving their outcomes.

A variety of healthcare professionals from across the UK were keen to share their local experiences (both good and bad). Among a variety of issues discussed was the importance of the multidisciplinary team, specialist heart failure teams and dedicated heart failure units. Challenges consistent in all units UK-wide include the pressure on beds, with many patients receiving care from nonspecialists with limited access to echocardiography, significant delays in investigation and suboptimal treatment and follow-up on discharge. Dr Rob Howlett (GPSI, Thaxted, Essex, UK) emphasized the need for good communication with patients and finished with a recorded interview of a patient reflecting on his own, often unsatisfactory, experiences. This provided a strong reminder of the need for us all to strive to do better in this area.

\section{The challenge of comorbidities}

A recurrent theme throughout the symposium was the consideration that heart failure patients admitted to hospital are often elderly, have a number of comorbidities and are not always treated on cardiology wards (by cardiologists). The importance of looking after our patients, regardless of the primary reason for admission (whether it be a complication of heart failure - or its treatment - or an unrelated comorbidity) was presented by Dr John Baxter (Sunderland Royal Hospital, UK). In an entertaining yet powerful presentation, he emphasized the need to view heart failure patients holistically and the importance of seeking to provide specialist input to patients with complex comorbidity who may not be conveniently situated on a cardiology ward.

\section{The Philip Poole-Wilson lecture}

The Philip Poole-Wilson lecture was given by Professor Sian Harding (Imperial College, London, UK). She, as one of the pioneers in this field, shared fascinating insights into the history and development of $\beta$-blocker therapy

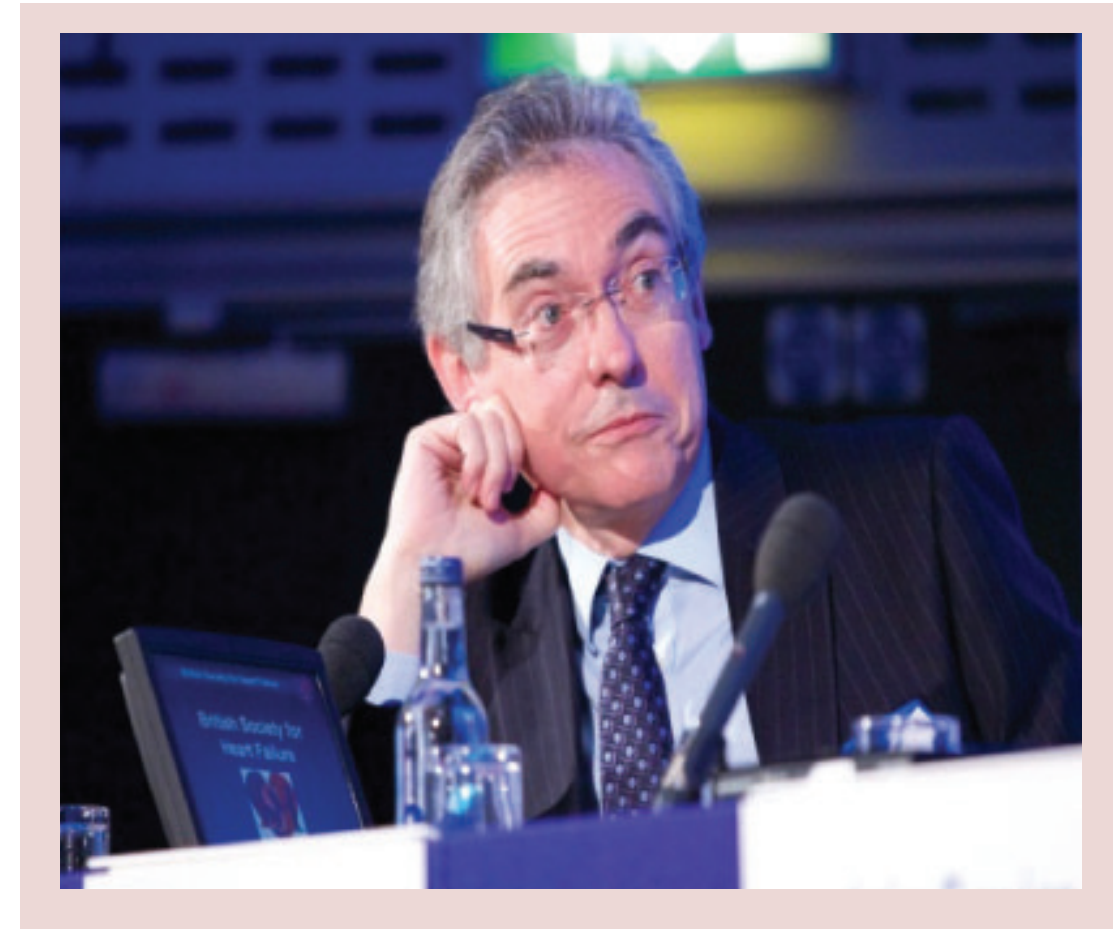

Figure 2. Professor John Cleland (Imperial College, London, UK).

in the treatment of heart failure (Figure 4). Not only did her talk provide an excellent example of how research has shaped and changed, the clinical trajectory of heart failure management to date, it also served as a reminder of the importance of ongoing basic science, translational and clinical research. Following this, we heard three abstract presentations by cardiologists

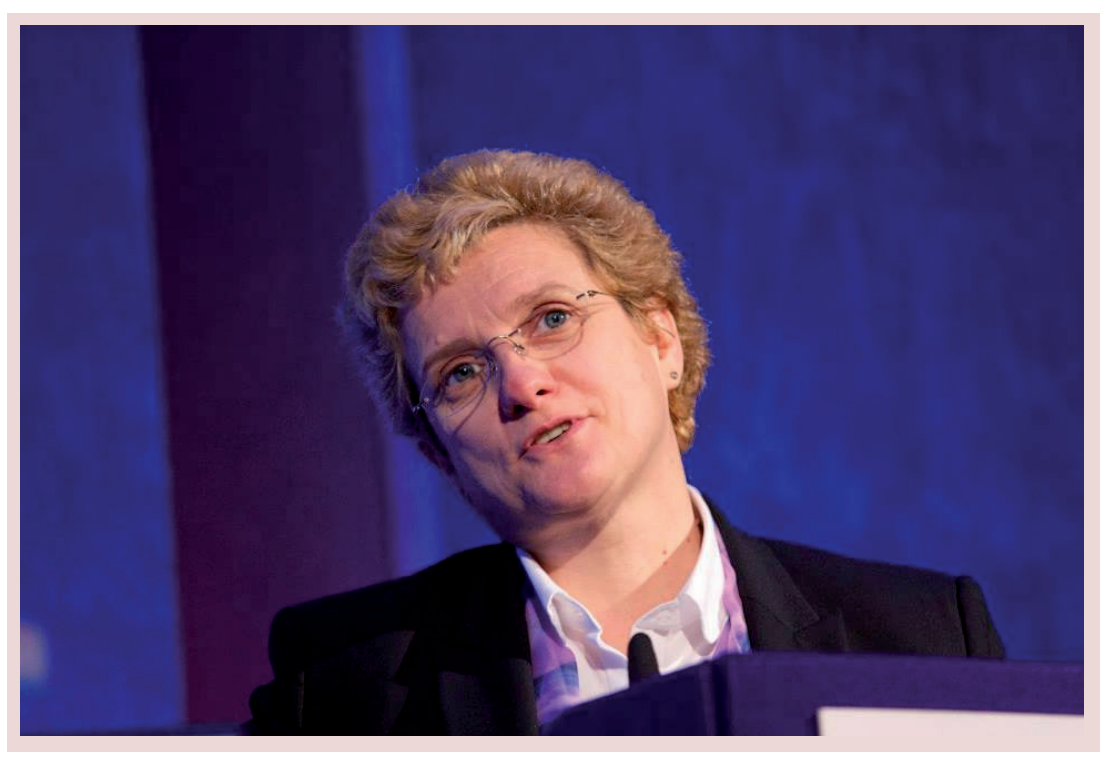

Figure 3. Theresa McDonagh (King's College, London, UK). 


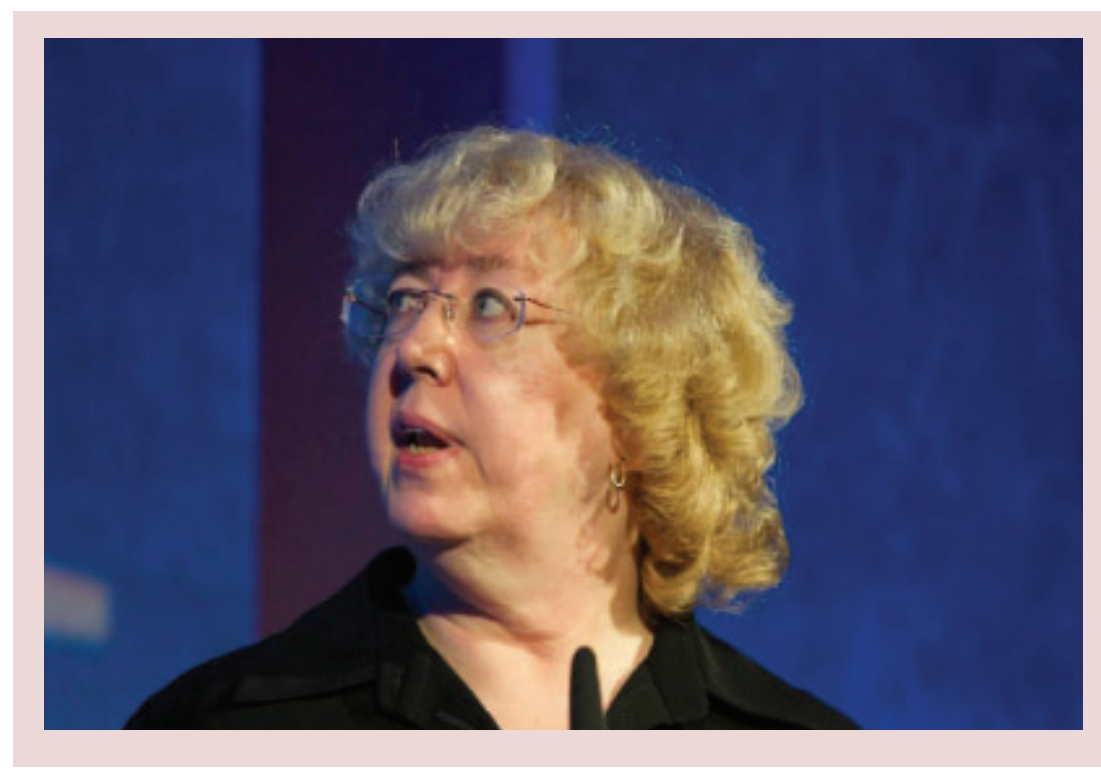

Figure 4. Professor Sian Harding (National Heart \& Lung Institute, London, UK).

in training as part of the Young Investigators Award and the announcement of the inaugural British Society of Heart Failure fellow award (supported by Servier).

\section{Heart failure with preserved ejection fraction}

In some series, heart failure with preserved ejection fraction (HF-PEF) accounts for more than $50 \%$ of all heart failure cases. Although

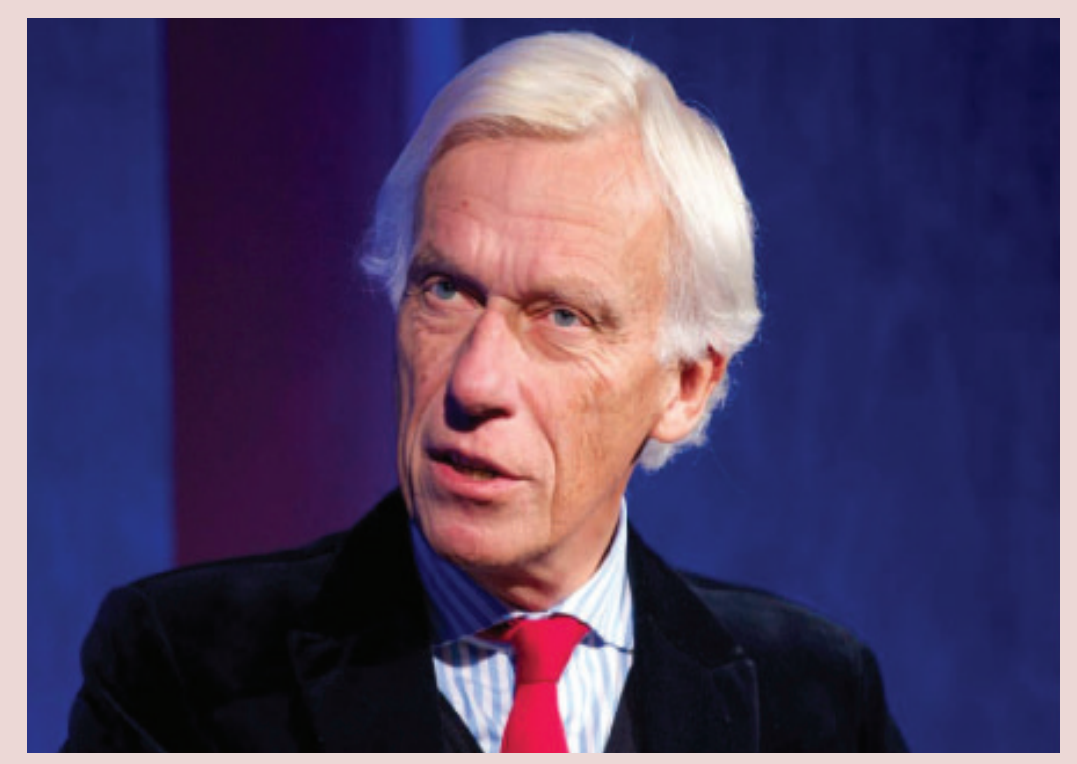

Figure 5. Professor Walter Paulus (VU University Medical Centre, Amsterdam, The Netherlands). progress has been made in identifying a new paradigm for HF-PEF development (beginning with a systemic proinflammatory state leading to hypertrophy, interstitial fibrosis and ultimately high diastolic left ventricular stiffness) no treatment has been shown, convincingly, to reduce morbidity and mortality in patients with HF-PEF. The second keynote lecture by Professor Walter Paulus (VU University Medical Centre, Amsterdam, The Netherlands) reviewed the pathophysiology of HF-PEF and concluded that HF-REF and HF-PEF are distinct heart failure phenotypes (Figure 5).

\section{Other talks in brief}

Other topics covered at the meeting included cardiac amyloid (Dr Carol Whelan, Royal Free Hospital, London, UK), peripartum cardiomyopathy (Dr Mark Petrie, Golden Jubilee Hospital, Glasgow, UK), treatment options in diuretic resistance (Dr Martin Thomas, The Heart Hospital, London, UK) and updates on various device therapies in heart failure patients; specifically, cardiac resynchronization therapy, implantable cardioverter-defibrillators and left ventricular-assist device implantation given by Dr Peter Cowburn (Southampton University Hospitals NHS Trust, UK), Dr Derek Connelly (Golden Jubilee National Hospital) and Dr Steve Shaw (Wythenshawe Hospital, Manchester, $\mathrm{UK})$, respectively.

Future British Society of Heart Failure meetings

- Heart Failure Day for Training and Revalidation, Beardmore Hotel and Conference Centre, Clydebank, Glasgow, 20 March 2014

- Heart Failure Nurse Study Day, Beardmore Hotel and Conference Centre, Clydebank, Glasgow, 21 March 2014

- 17th Annual Autumn Meeting, London, 27-28 November 2014

Financial \& competing interests disclosure The authors have no relevant affiliations or financial involvement with any organization or entity with a financial interest in or financial conflict with the subject matter or materials discussed in the manuscript. This includes employment, consultancies, honoraria, stock ownership or options, expert testimony, grants or patents received or pending, or royalties.

No writing assistance was utilized in the production of this manuscript. 